\title{
In Silico Investigation of the CACNA1C N2091S Mutation in Timothy Syndrome
}

\author{
Jieyun Bai ${ }^{1}$, Yaosheng Lu${ }^{1}$, Tao Song ${ }^{2}$, Kuanquan Wang ${ }^{3}$, Henggui Zhang ${ }^{3,4}$ \\ ${ }^{1}$ Department of Electronic Engineering, College of Information Science and Technology, Jinan \\ University, Guangzhou, China \\ ${ }^{2}$ Department of Cardiology, First Affiliated Hospital of Harbin Medical University, Harbin, China \\ ${ }^{3}$ School of Computer Science and Technology, Harbin Institute of Technology, Harbin, China \\ ${ }^{4}$ Biological Physics Group, School of Physics and Astronomy, University of Manchester, \\ Manchester, UK
}

\begin{abstract}
Experimental studies demonstrated that CACNA1CN2091S led to a gain-of-function in the L-type calcium current $\left(I_{\text {CaL }}\right)$ linked to heritable Timothy Syndrome, but mechanisms by which the N2091S mutation promotes and perpetuates ventricular fibrillation remain unclear. This study sought to investigate the proarrhythmic effects of N2091S-induced $I_{\text {CaL }}$. Using a dynamic ventricular myocyte model, we simulated $I_{C a L}, A P s, C^{2+}$ transients $\left(\left[\mathrm{Ca}^{2+}\right]_{i}\right)$ and sarcoplasmic reticulum (SR) calcium profiles $\left(\left[\mathrm{Ca}^{2+}\right]_{S R}\right)$ in three cell types. Effects of the N2091S mutation on cell electrophysiology were quantified by changes in $\mathrm{I}_{\mathrm{CaL}}$ density, $\left[\mathrm{Ca}^{2+}\right]_{i}$ amplitude $\left(\left[\mathrm{Ca}^{2+}\right]_{i(\max )}\right)$, SR calcium content $\left(\left[\mathrm{Ca}^{2+}\right]_{S R(\max )}\right)$, action potential duration (APD) and AP shape. It was shown that the N2091S mutation increased $I_{C a L(\max )},\left[\mathrm{Ca}^{2+}\right]_{i(\max )},\left[\mathrm{Ca}^{2+}\right]_{S R(\max )}$ and $A P D$ in three cell types. Compared with ENDO and EPI cells, MCELL cells with excessive prolongation of APD due to the N2091S mutation facilitated inducibility of early afterdepolarization (EAD)-mediated triggered activity. And the different EAD inducibility among the three cell types can amplify the electrical difference and thereby dispersion of repolarization, increasing susceptibility to ventricular arrhythmias. Thus, the N2091S mutation confers not only a trigger, but also a substrate for lethal ventricular arrhythmias.
\end{abstract}

\section{Introduction}

Timothy syndrome (TS) is a rare disorder that affects many parts of the body including the heart, digits (fingers and toes), and the nervous system[1]. TS is caused by gainof-function mutations of the CACNAIC gene, which encodes the L-type $\mathrm{Ca}^{2+}$ channel, $\mathrm{Ca}_{\mathrm{v}} 1.2$, and is characterized by QT prolongation, congenital heart disease, cognitive and behavioral problems[2].

Cav1.2 plays an important role in regulating excitationcontraction coupling and modulating cellular excitability
[3]. Abnormalities in Cav1.2 due to gain-of-function mutations of the CACNAIC gene have been suggested as factors contributing to ventricular arrhythmogenesis[4-6]. Recently, Sutphin et al. identified the variant p.N2091S (c.6272A $>$ G) and functional analysis revealed the N2091S variant led to a $105 \%$ gain-of-function in the L-type calcium current $\left(I_{C a L}\right)$ and minor kinetic alterations including a $-3.4 \mathrm{mV}$ shift in the half-maximal voltage of activation $\left(V_{a, 0.5}\right)[7]$. However, the ionic mechanisms by which the CACNA1C-N2091S mutation promotes and perpetuates ventricular fibrillation remain unclear.

In this study, changes in $I_{C a L}$ channel kinetics arising from the CACNA1C N2091S mutation were incorporated into the dynamic ventricular myocyte models for representing endocardial (ENDO), middle (MCELL) and epicardial (EPI) cells. Using the validated cell models, we investigated its effects on action potential (AP) and calcium transient $\left(\left[\mathrm{Ca}^{2+}\right]_{i}\right)$. Changes in $I_{C a L}$, AP profile, AP duration (APD), $\left[\mathrm{Ca}^{2+}\right]_{\mathrm{i}}$, sarcoplasmic reticulum calcium content $\left(\left[\mathrm{Ca}^{2+}\right]_{\mathrm{SR}}\right)$ and activation fraction of $\mathrm{Ca}^{2+} /$ calmodulin-dependent protein kinase II ( CaMKII $\left.I_{\text {active }}\right)$ were quantified to analyze pro-arrhythmic effects of the $C A C N A 1 C \mathrm{~N} 2091 \mathrm{~S}$ mutation.

\section{Methods}

The well-known TP06 model for human ventricular myocytes was modified to incorporate CaMKII regulation in our previous study. Some modifications to the modified TP06 model were included to reproduce $I_{C a L}$ channel kinetics arising from the $C A C N A 1 C$ N2091S mutation. In the cellular model, an ordinary differential equation was used to describe the transmembrane potential $V$ :

$$
\frac{d V}{d t}=-\frac{I_{\text {ion }}}{C_{m}}
$$

where $t$ is the time, $C_{m}$ is the capacitance across the cell membrane and $I_{i o n}$ is the total ionic current across the membrane. $I_{i o n}$ is given by

$$
I_{\text {ion }}=I_{N a}+I_{C a L}+I_{t o}+I_{K r}+I_{K s}+I_{K 1}+I_{N C X}+
$$




$$
I_{N a K}+I_{p K}+I_{p C a}+I_{N a b}+I_{C a b}
$$
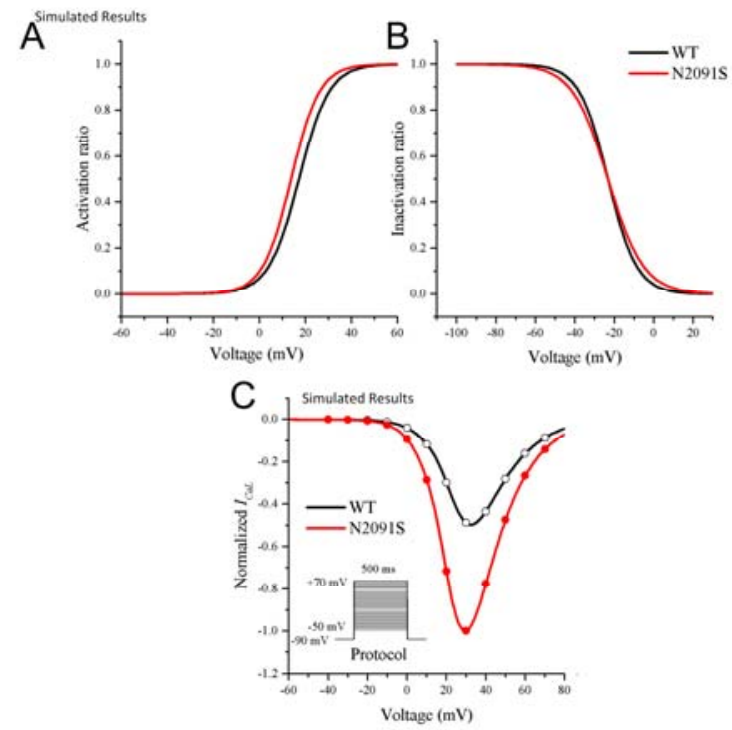

Figure 1. Functional $\mathrm{Ca}_{v} 1.2$ in human ventricular cells. Activation (A), inactivation (B) and current-voltage (C) curves under wild-type (WT) and N2091S Cav1.2 conditions.

In the cellular model, we determined the modifications to the original $I_{C a L}$ model to reproduce the behavior of the mutant $I_{C a L}$ during the same voltage-clamp employed in experiments[7]. Theoretical steady-state activation and inactivation curves which were used to simulate WT and N2091S $I_{C a L}$, are shown in Figure 1A\&B. Second, based on current-voltage relationships, mathematical models of $I_{C a L}$ were constructed (Figure 1C). This was achieved by simulating the experimental voltage-clamp protocol and scaling relative current proportions. CACNAIC mutationsinduced changes in $I_{C a L}$ channel kinetics include $V_{a, 0.5}$ the half-activation voltage, $S_{a}$ the slope of the steady-state activation of $I_{C a L}$ channel, $V_{\text {ina, } 0.5}$ the half-inactivation voltage, $S_{\text {ina }}$ the slope of the steady-state inactivation of $I_{C a L}$ channel, and $C S F$ a scaling factor of the maximal conductance of $I_{C a L}$ (for details see Table 1).

Table 1. The N2091S mutation-induced changes in $I_{\mathrm{CaL}}$ channel kinetics.

\begin{tabular}{ccc}
\hline Parameters & WT & N2091S \\
\hline$C S F$ & 1 & 1.73 \\
$V_{a, 0.5}(\mathrm{mV})$ & 6.6 & 6.2 \\
$S_{a}$ & -23.3 & -23.3 \\
$V_{\text {ina }, 0.5}(\mathrm{mV})$ & 7.35 & 9.09 \\
$S_{\text {ina }}$ & 6.6 & 6.2 \\
\hline
\end{tabular}

$$
\begin{gathered}
I_{C a L} \text { is described as } \\
I_{C a L}=C S F G_{C a L} d f f_{2} 4 \frac{(V-15) F^{2}}{R T} \frac{0.25 C a_{S S} e^{\frac{2(V-15) F}{R T}}-C a_{o}}{e^{\frac{2(V-15) F}{R T}}-1} \\
\qquad \frac{d d}{d t}=\frac{\left(d_{\infty}-d\right)}{\tau_{d}}
\end{gathered}
$$

$$
\begin{aligned}
& \boldsymbol{d}_{\infty}=\frac{1}{1+e^{\frac{V_{a, 0.5}-V}{S_{a}}}} \\
& \frac{\boldsymbol{d} \boldsymbol{f}}{\boldsymbol{d} \boldsymbol{t}}=\frac{\left(\boldsymbol{f}_{\infty}-\boldsymbol{f}\right)}{\boldsymbol{\tau}_{\boldsymbol{f}}} \\
& f_{\infty}=\frac{1}{1+e^{\frac{V-V_{\text {ina }, 0.5}}{S_{\text {in } a}}}}
\end{aligned}
$$

where $G_{C a L}$ is the maximal conductance of $I_{C a L}(\mu \mathrm{S} / \mathrm{pF}), d$ is the activation variable, $f$ is the voltage-dependent inactivation variable and $\tau_{f}$ is the voltage-dependent time constant of inactivation.

\section{Results}

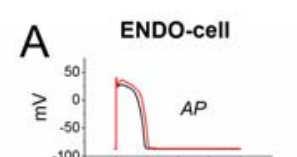

B
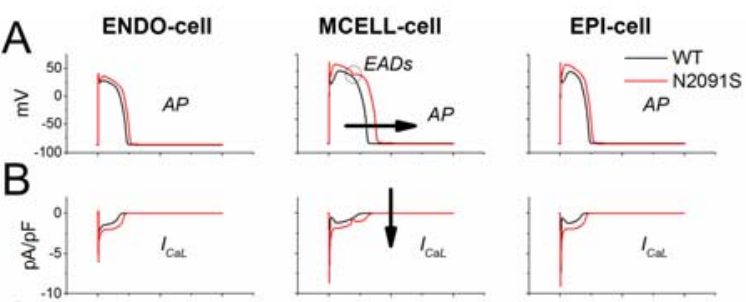

C
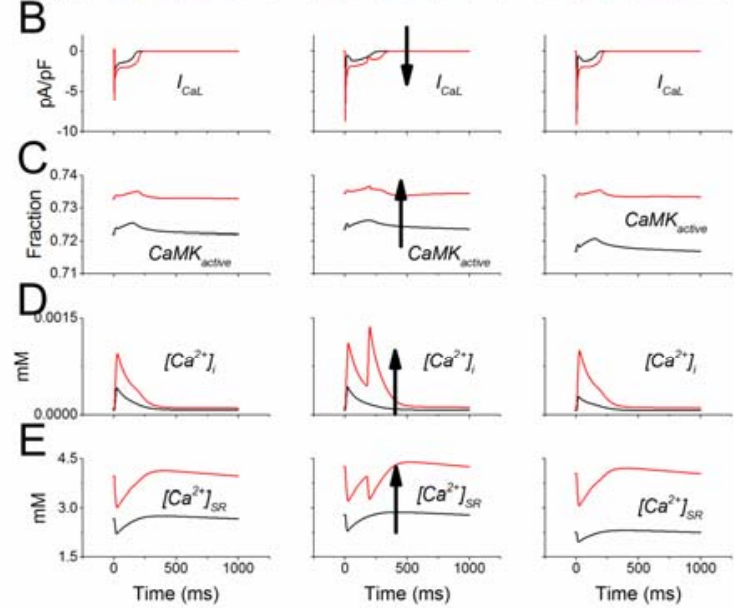

Figure 2. Comparison of action potentials and calcium transients between wild-type (WT) and N2091S Cav1.2 conditions in endocardial (ENDO), middle (MCELL) and epicardial (EPI) cells. AP (A), $I_{\text {CaL }}(\mathbf{B})$, CaMKII $_{\text {active }}(\mathbf{C})$, $\left[\mathrm{Ca}^{2+}\right]_{\mathrm{i}}(\mathbf{D})$ and $\left[\mathrm{Ca}^{2+}\right]_{\mathrm{SR}}(\mathbf{E})$.

$I_{C a L}$ arising from the Cav1.2 mutations altered intracellular calcium handling and prolonged $\mathrm{AP}$ as shown in Figure 2. For ENDO cells, the $I_{C a L}$ amplitude was increased by the CACNA1C N2091S mutation. Increased inward $\mathrm{Ca}^{2+}$ highly activated CaMKII, increased $\left[\mathrm{Ca}^{2+}\right]$, led to SR calcium loading and prolonged APD. In details, the $I_{C a L}$ amplitude was increased from $-4.74 \mathrm{pA} / \mathrm{pF}$ under the WT condition to $-6.06 \mathrm{pA} / \mathrm{pF}$. CaMKII active were 0.725 and 0.735 , under WT and N2091S conditions, respectively. The N2091S mutation caused an increase by $128 \%$ in $\left[\mathrm{Ca}^{2+}\right]_{\mathrm{i}}(4.15 \mathrm{E}-4 \mathrm{mM}$ under the WT condition vs. $9.5 \mathrm{E}-4$ $\mathrm{mM}$ under the $\mathrm{N} 2091 \mathrm{~S}$ condition). SR calcium overloading 
( $\left[\mathrm{Ca}^{2+}\right]_{\mathrm{SR}}$ was increased from $2.76096 \mathrm{mM}$ to $\left.4.13024 \mathrm{mM}\right)$ was observed under the N2091S condition. The measured APD was prolonged by this mutation from $242 \mathrm{~ms}$ to 292 ms.

In addition, the transmural electrical heterogeneity was investigated by producing APs of ENDO, MCELL, and EPI cells. In the three cells, the extent of changes in AP characteristics was most for MCELL cells (for details see Table 2). Importantly, under the N2091S condition, an early afterdepolarization (EAD) in the MCELL cells was induced, but no ectopic beats in the EPI and ENDO cells were observed.

Table 2. Electrophysiological characteristics (including $I_{\text {CaL }}, C a M K I I_{\text {active, }},\left[\mathrm{Ca}^{2+}\right]_{\mathrm{i}}, \quad\left[\mathrm{Ca}^{2+}\right]_{\mathrm{SR}}$ and $\left.\mathrm{APD}_{90}\right)$ of ventricular cells under wild-type (WT) and N2091S Cav1.2 conditions.

\begin{tabular}{cccccc}
\hline & $\begin{array}{c}I_{C a L} \\
(\mathrm{pA} / \mathrm{pF})\end{array}$ & $\begin{array}{c}\text { CaMK } \\
I_{\text {active }}\end{array}$ & $\begin{array}{c}{\left[\mathrm{Ca}^{2+}\right]_{\mathrm{i}}} \\
(\mathrm{mM})\end{array}$ & $\begin{array}{c}{\left[\mathrm{Ca}^{2+}\right] \mathrm{SR}} \\
(\mathrm{mM})\end{array}$ & $\begin{array}{c}\mathrm{APD} 90 \\
(\mathrm{~ms})\end{array}$ \\
\hline $\begin{array}{c}\mathrm{ENDO} \\
(\mathrm{WT})\end{array}$ & -4.74 & 0.725 & $4.15 \mathrm{E}-4$ & 2.76096 & 242 \\
$\begin{array}{c}\mathrm{MCELL} \\
(\mathrm{WT})\end{array}$ & -7.64 & 0.726 & $4.33 \mathrm{E}-4$ & 2.88162 & 318 \\
$\begin{array}{c}\mathrm{EPI} \\
(\mathrm{WT})\end{array}$ & -8.07 & 0.721 & $2.88 \mathrm{E}-4$ & 2.32031 & 243 \\
$\begin{array}{c}\text { ENDO } \\
(\mathrm{N} 2091 \mathrm{~S})\end{array}$ & -6.06 & 0.735 & $9.5 \mathrm{E}-4$ & 4.13024 & 292 \\
$\begin{array}{c}\text { MCELL } \\
(\mathbf{N 2 0 9 1 S})\end{array}$ & $\mathbf{- 8 . 7 2}$ & $\mathbf{0 . 7 3 7}$ & $\mathbf{1 . 3 6 E - 3}$ & $\mathbf{4 . 3 9 8 9 8}$ & $\mathbf{4 2 9}$ \\
$\begin{array}{c}\text { EPI } \\
(\mathrm{N} 2091 \mathrm{~S})\end{array}$ & $\mathbf{- 9 . 0 9}$ & 0.735 & $9.98 \mathrm{E}-4$ & 4.21031 & 295 \\
\hline
\end{tabular}

\section{Discussion}

This study investigated effects of the CACNAIC N2091S mutation on ventricular electrical activity at the cellular level. Our main findings are as follows: (1) Increased $I_{C a L}$ arising from the CACNA1C N2091S mutation prolonged APD and caused calcium overload, (2) Increased $\mathrm{Ca}^{2+}$ ions due to the CACNA1C N2091S mutation highly activated CaMKII and elevated $\left[\mathrm{Ca}^{2+}\right]_{\mathrm{i}}$ and $\left[\mathrm{Ca}^{2+}\right]_{\mathrm{SR}}$, resulting in spontaneous $\mathrm{SR}$ calcium releases during the phase 3 of the AP and thereby EADs, (3) this mutation increased the intrinsic transmural heterogeneity, and (4) the vulnerability of MCELL cell to EADs was increased by this mutation. Consequently, these findings demonstrate that the CACNAIC N2091S mutation increases the likelihood of ventricuar arrhythmias due to APD prolongation and triggered activity, which facilitate complex ventricular contractions.

Computational modeling has shed valuable light on the ionic mechanisms underlying complex ventricular contractions. Gain-of-function muations, which include G406R [8], G402S\&G406R [9] and R858H [10], were associated with APD prolongation, intracellular calcium handling abnormalities and triggered activity. In additon, alterations in calcium signaling (CaMKII) due to increased
$\left[\mathrm{Ca}^{2+}\right]_{\mathrm{i}}$ arising from these mutation also partly contritubed to triggered activity[11]. In this study, the cellular mechanism is that $I_{C a L}$ due to the N2091S mutation prolongs APD, activates CaMKII and increases SR calcium load, leading to EADs. These simulation data of the present study add to the growing weight of evidence implicating cellular mechanisms of CACNA1C mutations.

\section{Conclusion}

Thus, these human ventricular models provide powerful tools for drug screening and investigating cardiac dynamics. Our findings of these simualted results suggest the triggered activity due to EADs arising from the CACNA1C-N2091S mutation may contribute to the mechanism underlying ventricular arrhythmias.

\section{Acknowledgements}

This work was supported by the National Natural Science Foundation of China (No. 61901192) (J.B.), the Science and Technology Planning Project of Guangdong Province (No.2015B020214004 and No.2015B020233010) (Y.L.), the National Key R\&D Program of China (No. 2017YFC0113000), the National Natural Science Foundation of China (No: 61571165 and 61572152) (K.W.) and Science Technology and Innovation Commission of Shenzhen Municipality (No: JCYJ20151029173639477 and JSGG20160229125049615) (H.Z.).

\section{References}

1. Splawski, I., et al., Cav1. 2 calcium channel dysfunction causes a multisystem disorder including arrhythmia and autism. Cell, 2004. 119(1): p. 19-31.

2. Bai, J., et al., Pro-arrhythmogenic effects of CACNA1C G1911R mutation in human ventricular tachycardia: insights from cardiac multi-scale models. Scientific reports, 2016. 6: p. 31262.

3. Bers, D., Cardiac excitation-contraction coupling. Nature, 2002. 415(6868): p. 198-205.

4. Boczek, N.J., et al., Novel Timothy syndrome mutation leading to increase in CACNA1C window current. Heart rhythm, 2015. 12(1): p. 211-219.

5. Boczek, N.J., et al., Exome sequencing and systems biology converge to identify novel mutations in the L-type calcium channel, 
CACNA1C, linked to autosomal dominant long QT syndrome. Circulation: Cardiovascular Genetics, 2013. 6(3): p. 279-289.

6. Boczek, N.J., et al., Identification and functional characterization of a novel CACNA1C-mediated cardiac disorder characterized by prolonged $Q T$ intervals with hypertrophic cardiomyopathy, congenital heart defects, and sudden cardiac death. Circulation: Arrhythmia and Electrophysiology, 2015. 8(5): p. 1122-1132.

7. Sutphin, B.S., et al., Molecular and functional characterization of rare CACNAIC variants in sudden unexplained death in the young. Congenital heart disease, 2016. 11(6): p. 683-692.

8. Splawski, I., et al., Severe arrhythmia disorder caused by cardiac L-type calcium channel mutations. Proceedings of the National Academy

Address for correspondence:

Dr. Jieyun Bai

Department of Electronic Engineering

College of Information Science and Technology

Jinan University, Guangzhou, China

E-mail: bai_jieyun@126.com of Sciences, 2005. 102(23): p. 8089-8096.

9. $\quad$ Sung, R.J., et al., $\beta$-Adrenergic modulation of arrhythmogenesis and identification of targeted sites of antiarrhythmic therapy in Timothy (LQT8) syndrome: a theoretical study. American Journal of Physiology-Heart and Circulatory Physiology, 2009. 298(1): p. H33-H44.

10. Bai, J., et al., Computational cardiac modeling reveals mechanisms of ventricular arrhythmogenesis in long QT syndrome type 8: CACNA1C R858H mutation linked to ventricular fibrillation. Frontiers in physiology, 2017. 8: p. 771.

11. Thiel, W., et al., Proarrhythmic defects in Timothy syndrome require calmodulin kinase II. Circulation, 2008. 118(22): p. 2225-2234. 